\title{
A Non-parametric Probabilistic Model for Hepatic Tumor Detection
}

\author{
Y. Konno, X.H. Han, Y.W. Chen \\ Graduate School of Information Science and Engineering \\ Ritsumeikan University \\ Shiga, Japan
}

Abstract-Automatic hepatic tumor enhancement and detection in CT volume data is an important preprocessing step in computer-aided diagnosis of liver tumor. In this paper, we proposed a novel non-parametric probabilistic model for automatic tumor detection. Compared with conventional method such as Gaussian mixture model, our proposed method is parameter-free method and can be applied to abnormal livers even with different type tumors. The proposed method is easy to implement and the computation cost is also low compared with other method.

Keywords-non-parametric; probability model; gaussian mixture model; tumor detection

\section{INTRODUCTION}

Liver cancer is considered as a major death factor in Japan. In 2012, number of fatality by liver cancer is the 4th most common cause of death by cancer [1]. The survival rate of the early stage 1 is $50.2 \%$, while those of stage 3 and the last stage 4 are $14.5 \%$ and $6.0 \%$, respectively. So tumor detection in early stage is essential for increasing the survival chances of patients. Medical images, such as CT are used as a non-invasive method to detect tumors (malignant tumors are called cancerous tumors). Recent advancements in medical imaging modalities have enabled the acquisition of high-resolution CT datasets, so that a physician can detect small tumors, in addition to large tumors. Owing to the large number of images in medical datasets, it is difficult to manually analyze all images, and useful diagnostic information may be overlooked. Moreover, the diagnoses are mainly based on the physician's subjective evaluation and are dependent on the physician's experience. Therefore, automatic tumor enhancement and detection in CT volumes based on computer vision techniques, which is also known as computer aided detection (CAD), has become one of the major research subjects in the field of medical image analysis.

Until now, many methods have been proposed for tumor enhancement and detection in liver CT images. These methods can be classified as semi-automatic [2][3] and automatic [4][5]. Smeets et al. have proposed a semi-automatic level set method, which combines a spiral scanning technique with supervised fuzzy pixel classification [2]. Mala et al. employed waveletbased texture features in order to train a neural network for use in tumor detection [4]. In the method proposed by Park et al. [5], vessels are removed from liver images and a bimodal histogram is assumed for the intensity distribution of the liver and tumors. The optimal threshold to segment tumors is determined by a "mixture density" algorithm. Previous study [6], they proposed a tumor detection technique based on the

\author{
X. Wei \\ Institute for Infocomm Research \\ A-STAR, Singapore
}

Expectation Maximization/ Maximization of Posterior Marginals (EM/MPM) [7].

All the above mentioned methods can locate tumors that are sufficiently large and have distinct boundaries. Semi-automatic approaches for handling a large number of tumors would need extensive user interactions, and therefore are error prone and tedious. In this paper, we propose a novel hepatic tumor enhancement and detection strategy by extracting the existing probability of the tumor tissue compared to the liver and vessel ones in CT volumes. As we know that the healthy liver and vessel tissues in any CT volume is generally existed in all slices, and then, is easily obtained as prototypes of common tissues. However, due to large variations and impossible to achieving the adaptive tumor tissue for a specific patient, the tumor prototype is extremely difficult for extraction. Therefore, this paper explores to only sample the liver and vessel tissues as common prototype, and then calculate the existing probability of uncommon tissues such as tumor based on the collected prototypes of common tissues by applying a non-parametric framework. The proposed strategy is composed of 4 steps: (1) automatic segment the liver region from the input CT volume using a K-means clustering and geodesic active contour algorithms [8]; (2) obtain some prototypes of the healthy liver tissues by sampling the segmented liver regions in a tumor-free region defined by the user; (3)calculate the existing probability of tumor tissue using a non-parametric framework; (4) detect tumor candidates by a defined threshold. The procedure of the proposed strategy is shown in Fig. 1. The remaining parts of this paper are prepared as follows. In section 2, related work is reviewed. Section 3 describes the proposed method. Section 4 shows experimental results and 5 concludes the paper.

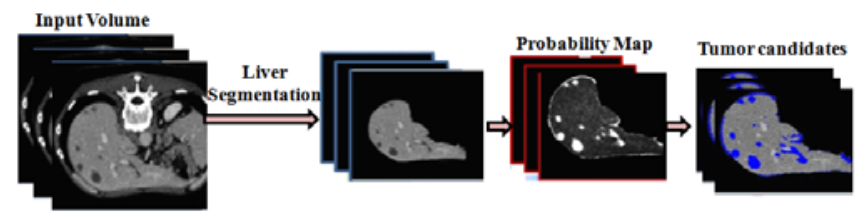

FIGURE I. THE FLOWCHART OF THE PROPOSED NONPARAMETRIC PROBABILISTIC MODEL FOR HEPATIC TUMOR ENHANCEMENT IN CT VOLUMES.

\section{RELATED WORK}

As shown in Figure 2, we can see that the intensity of vessel tissues is the highest in three, while the intensity of tumors is the lowest. So most conventional tumor detection methods use intensity information. 
One of typical methods is to use a Gaussian Mixture Model (GMM) to represent the intensity distribution (histogram). In the method proposed by Park et al. [5], vessels are removed from liver images and a bimodal histogram (a Gaussian mixture model with two components) is assumed for the intensity distribution of the pure liver and tumors. The Expectation Maximization (EM) algorithm can be used to find model parameters.

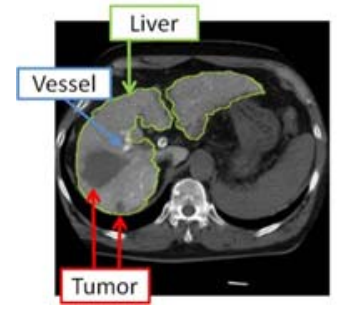

FIGURE II. (LEFT): TYPICAL CT IMAGE WITH AN ABNORMAL LIVER

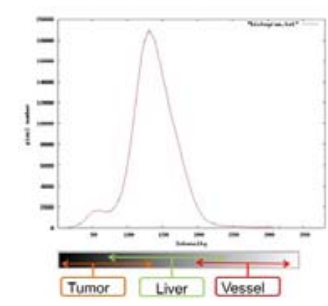

FIGURE III. (RIGHT): INTENSITY DISTRIBUTION OF AN ABNORMAL LIVER

There are three limitations in the conventional GMM based method. The first limitation is that the method is sensitive to the initial parameter. The second limitation is that we need to determine the number of components. Since the different type of tumor will have different intensity range. Since EM is an iteration method, it may take large computation cost for tumor detection.

\section{TUMOR DETECTION USING NON-PARAMETRIC PROBABILISTIC MODEL}

In this paper, we propose a novel non-parametric probabilistic model for tumor detection in order to improve the detection accuracy and reduce the computation cost. The basic idea is that we use normal liver volumes to extract prototypes of the pure liver and the vessel and then we propose a nonparametric probabilistic model, which is based on the similarity of input volume patch and the prototypes, to calculate the probability of the tumor for each voxel. The transformed probability map (the intensity of each voxel is the probability of the tumor) is used for detection.

\section{A. Probabilistic Model Construction}

We randomly selected a normal liver CT volume. We first manually segment the liver volume from the CT volume. We then choose a 3D template with a size of $35 \times 35 \times 3$ from the liver volume randomly. Each voxel in the template (except the voxel on the boundary) is used to form a prototype vector with its 26 surrounding voxels. The dimension of the prototype is 27 $\times 1$, Figure 4 shows an example of prototype generation (2D case).

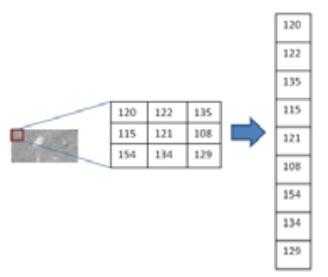

FIGURE IV. EXAMPLE OF PROTOTYPE GENERATION (2D CASE).

\section{B. Non-parametric Probabilistic Model}

In this paper, we propose a novel non-parametric probability model, which is based on the similarity of the input volume patch $\left(\mathbf{y}_{i}\right)$ and the prototypes $(\mathbf{x})$. We first calculate the distance of the input volume patch $(3 \times 3 \times 3)$ and all the prototype and then we choose $\boldsymbol{K}$ prototypes with the shortest distance for probability calculations. Equation (1) is our defined posterior probability of the pure liver and the vessel ( $p($ liver, vessel $\mid \mathbf{y})$ ).

$\mathbf{y}_{i}$ : The $i$ - thinput volume patch $\quad, \quad \mathbf{x}_{k}$ : The k - th prototype
$P\left(\right.$ Liver, Tumor $\left.\mid \mathbf{y}_{i}\right)=\frac{1}{K} \sum_{k=1}^{K}\left(\exp \left[-\frac{\left(\mathbf{y}_{i}-\mathbf{x}_{k}\right)^{T} \Sigma^{-1}\left(\mathbf{y}_{i}-\mathbf{x}_{k}\right)}{2}\right]\right)$

where $\Sigma$ is the co-variance matrix of the prototypes. Since medical volume usually contains noise, even the input volume patch ( $\mathbf{y})$ is the pure liver or the vessel, the distance of $\mathbf{y}$ and $\mathbf{x}$ is not zero. In order to take account of the effect of noise, we rewrite our probability model (Eq.(1)) as Eq.2).

$$
\begin{aligned}
& P\left(\text { Liver, vessel } \mid \mathbf{y}_{i}\right)=\frac{1}{K} \sum_{k=1}^{K}\left(\exp \left[-\frac{\max \left[0,\left(\mathbf{y}_{i}-\mathbf{x}_{k}\right)^{T} \Sigma^{-1}\left(\mathbf{y}_{i}-\mathbf{x}_{k}\right)-c^{2}\right]}{2}\right]\right) \\
& \text { where } \quad c=\min _{p, q(p \neq q)} d_{p q}
\end{aligned}
$$

$d_{p q}$ is the distance of the $p$-th prototype and the $q$-th prototype. The probability of the tumor (the class which is not the liver and the vessel) can be written as Eq.(3).

$$
P\left(\text { Tumor } \mid \mathbf{y}_{i}\right)=1-\frac{1}{K} \sum_{k=1}^{K}\left(\exp \left[-\frac{\max \left[0,\left(\mathbf{y}_{i}-\mathbf{x}_{k}\right)^{T} \Sigma^{-1}\left(\mathbf{y}_{i}-\mathbf{x}_{k}\right)-c^{2}\right]}{2}\right]\right)
$$

Since in the numerator of Eq.(3) (or Eq.(2)), the computation of inverse matrix and product of vector and matrix is time consuming. In order to simplify the computation, we assume that there is no correlation between each element of the prototype.

Thus, the covariance matrix can be simplified as Eq.(4).

$$
\Sigma \approx\left[\begin{array}{cccc}
\lambda_{11} & 0 & \cdots & 0 \\
0 & \lambda_{22} & \cdots & 0 \\
\vdots & \vdots & \ddots & 0 \\
0 & 0 & 0 & \lambda_{n n}
\end{array}\right]
$$

where $\lambda$ is the diagonal element of the covariance matrix and $\boldsymbol{n}$ is the dimension of the prototype. Then Eq.(3) can be rewritten as Eq.(5). 


$$
\begin{aligned}
& P\left(\text { Tumor } \mid \mathbf{y}_{i}\right)=1-\frac{1}{K} \sum_{k=1}^{K}\left(\exp \left[-\frac{\max \left[0,\left(\mathbf{y}_{i}-\mathbf{x}_{k}\right)^{T}\left(\mathbf{y}_{i}-\mathbf{x}_{k}\right)-c^{2}\right]}{2 \delta^{2}}\right]\right) \\
& \text { where } \quad \delta=\prod_{i=1}^{n} \lambda_{i i}
\end{aligned}
$$

\section{Tumor Detection}

In tumor detection phase, we calculate the probability of the tumor for each voxel using Eq.(11). Each voxel $y_{i}$ with its 26 surrounding voxels is treated as an input volume patch $\mathbf{y}_{i}$. The input liver volume can be transformed to a probability map of the tumor, in which the intensity of each voxel is the posterior probability of the tumor $\left(\mathrm{P}\left(\operatorname{tumor} \mid y_{i}\right)\right)$. Then we detect the tumor by thresholding the probability map. The threshold value is determined by several trials.

\section{EXPERIMENT}

In order to validate the efficiency of our proposed method, we applied our proposed method to abnormal CT volumes. The CT dataset is a public dataset belonged to the MICCAI Liver Tumor Segmentation Challenge 2008 [8].

The CT volume size is $512 \times 512$ and the spacing is $0.72 \mathrm{~mm} \times 0.72 \mathrm{~mm} \times 1 \mathrm{~mm}$.

As a preprocessing, we use an anisotropic diffusion filter to remove the noise contained in the CT volume.

Two typical slice images and detection results are shown in Figure 5, 6. Figure 5(a) is original CT liver slice image. Figure $5(\mathrm{~b})$ is the image after noise reduction. Figure 6(a) is the transformed probability map of the tumors. Figure 6(b) is the detection results by our proposed method (the threshold value is 0.3 ) and Figure 6(c) is the detection result by conventional GMM method.
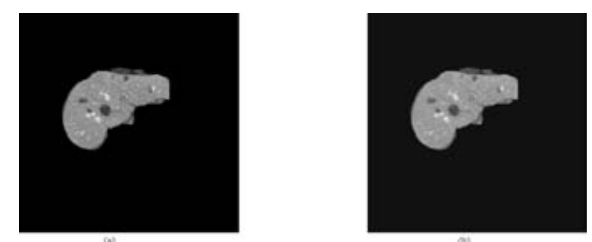

FIGURE V. ORIGINAL CT IMAGE(A) AND AFTER NOISE REDUCTION IMAGE(B).

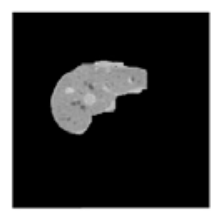

(a)

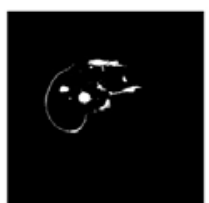

(b)

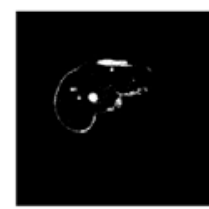

(c)
FIGURE VI. (A) PROBABILITY MAP OF THE TUMORS. (B) RESULTS BY OUR PROPOSED METHOD. (C) CONVENTIONAL GMM METHOD.

The detection results are summarized in Table 1, 2 .

It can be seen that our proposed method can detect all tumors with less false positive compared with conventional GMM method. Especially Table1, The recall and precision of our proposed method are $100 \%$ and $40.3 \%$, while the recall and precision of the conventional GMM method are $75 \%$ and
$2.26 \%$, respectively. In the conventional GMM method, the number of components is set as 3 (pure liver, vessel, and tumor).

The averaged intensity of true positive (detected tumors) is $72.9 \pm 22.7$, while the averaged intensity of false negative (not detected tumors) is 101.1 \pm 16.1 . We can see that the averaged intensity between the true positive and the false negative is quite different.

It means that different tumor may have different intensity.

It will be very difficult for conventional GMM method to detect the tumors with different intensity (we may need to set the number of components $\geq 3$ ). On the other hand, our proposed method do not need to set any parameters (parameter-free) and can detect tumors even with different intensity.

TABLE I. M23 TUMOR DETECTION RESULT.

\begin{tabular}{|c|c|c|c|c|}
\hline & FP & TP & Detection Total & Tumor Total \\
\cline { 1 - 4 } Conventional GMM & 1687 & 39 & 1726 & 50 \\
\cline { 1 - 4 } Our proposed method & 74 & 50 & 124 & \\
\hline
\end{tabular}

TABLE II. M27 TUMOR DETECTION RESULT.

\begin{tabular}{|c|c|c|c|c|}
\hline & FP & TP & Detection Total & Tumor Total \\
\cline { 1 - 4 } Conventional GMM & 680 & 3 & 683 & 3 \\
\cline { 1 - 4 } Our proposed method & 248 & 3 & 251 & \\
\hline
\end{tabular}

\section{CONCLUSION}

In this paper, we proposed a novel non-parametric probabilistic model for automatic tumor detection. Compared with conventional method such as Gaussian mixture model, our proposed method is parameter-free method and can be applied to abnormal livers even with different type tumors. The proposed method is easy to implement and the computation cost is also low compared with other existing method. Though in this paper, the liver is manually segmented from the CT volume in order to validate the detection accuracy, we can easily combine detection algorithm with some existing liver segmentation algorithms.

\section{ACKNOWLEDGEMENT}

This work is supported in part by the Grant-in Aid for Scientific Research from the Japanese Ministry for Education, Science, Culture and Sports (MEXT) under the Grant No. 2430076, No. 24103710, in part by the MEXT Support Program for the Strategic Research Foundation at Private Universities (2013-2017), and in part by the R-GIRO Research Fund from Ritsumeikan University.

\section{REFERENCES}

[1] Center for Cancer Control and Information Services, National Cancer Center, Japan, http://ganjoho.jp/professional/statistics/backnumber/2012.jp

[2] D. Smeets, D. Loeckx, B. Stijnen, B. De Dobbelaer, D. Vandermeulen, P. Suetens, "Semi-automatic level set segmentation of liver tumors combining a spiral scanning technique withsupervised fuzzy pixel classification", Medical image analysis, vol. 14, no. 1, pp. 13-20, February 2010. 
[3] Y. Hame, T. Alhonnoro. M., Pollari, “'Image Analysis for Liver Tumor Ablation Treatment Planning", Hands-on Image Processing 2009, Robotiker-Tecnalia.

[4] K. Mala, V. Sadasivam, S. Alagappan, '`Neural Network Based Texture Analysis of Liver Tumor from Computed Tomography Images", International Journal of Biomedical Sciences 2, 33-40, 2006.

[5] Seung-Jin Park, Kyung-Sik Seo, Jong-An Park: “Automatic Hepatic Tumor Segmentation Using Statical Optimal Threshold", Computational Science - ICCS 2005, Springer Berlin / Heidelberg, Volume 3514, pp 934-940, 2005.

[6] Y. Masuda, A. H. Foruzan, T. Tateyama, Y. W. Chen, “'Automatic liver tumor detection using EM/MPM algorithm and shape information", IEICE technical report 110(28), 25-30, 2010.

[7] Y. Masuda, A. H. Foruzan, T. Tateyama, Y. W. Chen, 'Liver Tumor Detection In CT Images by Adaptive Contrast Enhancement and The EM/MPM Algorithm", Proc. of IEEE Interntional Conference on Image Processing (ICIP2013), pp.1453-1456, 2011.

[8] X. Deng and G. Du, “Editorial: 3D Segmentation in the Clinic: A Grand Challenge II - Liver Tumor Segmentation", http://grandchallenge2008.bigr.nl/proceedings/liver/articles.html 